\title{
Volumetric modulated arc therapy for spine SBRT patients to reduce treatment time and intrafractional motion
}

\author{
Ahmad Amoush, Allison Dalton, Bryan Rabatic, Ke Huang, Ahmad Al-Basheer \\ Department of Radiation Oncology, Georgia Regents University, Augusta, Georgia, USA
}

Received January 05, 2015; Revised February 02, 2015; Accepted February 08, 2015; Published Online February 20, 2015

\section{Case Report}

\begin{abstract}
Volumetric modulated arc therapy (VMAT) is an efficient technique to reduce the treatment time and intrafractional motion to treat spine patients presented with severe back pain. Five patients treated with spine stereotactic body radiation therapy (SBRT) using 9 beams intensity modulated radiation therapy (IMRT) were retrospectively selected for this study. The patients were replanned using two arcs VMAT technique. The average mean dose was $104 \% \pm 1.2 \%$ and $104.1 \% \pm 1.0 \%$ in IMRT and VMAT, respectively $(\mathrm{p}=0.9)$. Accordingly, the average conformal index $(\mathrm{CI})$ was $1.3 \pm 0.1$ and $1.5 \pm 0.3$, respectively $(\mathrm{p}=0.5)$. The average dose gradient (DG) distance was $1.5 \pm 0.1 \mathrm{~cm}$ and $1.4 \pm 0.1 \mathrm{~cm}$, respectively $(\mathrm{p}=0.3)$. The average spinal cord maximum dose was $11.6 \pm 1.0 \mathrm{~Gy}$ and $11.8 \pm 1.1 \mathrm{~Gy}(\mathrm{p}=0.8)$ and $\mathrm{V}_{10 \mathrm{~Gy}}$ was $7.4 \pm 1.4 \mathrm{cc}$ and $8.6 \pm 1.7 \mathrm{cc}(\mathrm{p}=0.4)$ for IMRT and VMAT, respectively. Accordingly, the average number of monitor units (MUs) was $6771.7 \pm 1323.3 \mathrm{MU}$ and $3978 \pm 576.7 \mathrm{MU}$ respectively ( $\mathrm{p}=0.02$ ). The use of VMAT for spine SBRT patients with severe back pain can reduce the treatment time and intrafractional motion.
\end{abstract}

Keywords: VMAT; SBRT; Spine

\section{Introduction}

Spine stereotactic body radiation therapy (SBRT) delivers high ionization radiation to provide adequate dose to the target while sparing the surrounding normal structures. Doses usually ranges from 16 to $24 \mathrm{~Gy}$ in a single fraction or 24 to $35 \mathrm{~Gy}$ in 2 to 5 fractions. ${ }^{1}$ The tight planning margins and steep dose gradients require accurate targeting and immobilization of the target during irradiation. Patients with spinal metastasis usually present with a back pain and some neurologic problems..$^{2-3}$ The difficulty in applying radiosurgery for spinal patients is due to intrafractional motion associated with the pain. Even with a good immobilization technique, the patient can move during the treatment therefore shortening the irradiation time is an efficient factor to reduce the intrafractional motion.

Ryu et al. ${ }^{4}$ reported a precision for a given isocenter between simulation and actual treatment position of $1.36 \mathrm{~mm} \pm$ $0.11 \mathrm{~mm}$. Li et al. ${ }^{5}$ quantified the interfractional and intrafractional motion for spine SBRT patients using different immobilization devices. They reported the results based on cone-beam computed tomography (CBCTs) on initial setup residual error and the mid- and post- treatment CBCTs. For mid-treatment intrafractional motions, they reported standard deviation (SDs) of $<1 \mathrm{~mm}$ for vacuum fixation and S-frame cohorts but increased to $1.1 \mathrm{~mm}$ for evacuated cushion. For post-treatment, they reported intrafractional motion of $<1 \mathrm{~mm}$ for vacuum fixation and $1.3 \mathrm{~mm}$ for evac- uated cushion and S-frame groups. Maximum translational displacements at mid-treatment and post-treatment CBCTs were $3.4 \mathrm{~mm}$ in the lateral direction (vacuum fixation), and $4.5 \mathrm{~mm}$ in the lateral direction (evacuated cushion), respectively. These results suggested using the vacuum fixation to reduce the intrafractional motion. Also, shortening the treatment time will reduce the intrafractional motion especially when patient presents with severe back pain.

Volumetric modulated arc therapy (VMAT) is a rotational intensity modulated radiation therapy (IMRT) that can be delivered by varying the MLC leaf speed, dose rate and gantry speed. The advantage of VMAT when compared to the fixed field IMRT delivery is reduction of the treatment time while maintaining the similar, if not superior plan quality as the fixed field IMRT.6, 7,8 Matuszak et al. ${ }^{9}$ compared VMAT with fixed field IMRT for spine SBRT treatment and concluded that VMAT improved the isodose conformality and reduced the treatment time by $37 \%$. The Eclipse treatment planning system uses a 3D pencil beam superposition-convolution algorithm (AAA) for dose calculations. Rana et al. ${ }^{10}$ compared the AAA with Acuros XB (AXB) for lung SBRT and concluded that both algorithms satisfy the lung SBRT protocol. In this study, we are presenting five cases which were treated at our institution with SBRT for spine metastases using Intensity Modulated Radiation Therapy (IMRT). The cases were replanned using VMAT (Vari-

Corresponding author: Ahmad Amoush; Department of Radiation Oncology, Georgia Regents University, Augusta, Georgia, USA. 
an-RapidArc system) to study the reduction in treatment time, monitor units, and conformality index.

\section{Methods and Materials}

\section{Patient selection}

Five patients were retrospectively selected for this study. All patients were treated at our institution with spine SBRT using 9 fields IMRT plans. The treatment sites ranged from T5-L5 and the volume ranged from 25 to 100 cc (Table 1). Patients were treated following the recommendations of Radiation Therapy Oncology Group 0631 (RTOG-0631) ${ }^{11}$ and Task Group 101 of the American Association of Physicists in Medicine (AAPM-TG 101). ${ }^{12}$ Varian Trilogy and 23IX linear accelerators (Varian Medical systems, Palo Alto, CA) were used to deliver radiation. Only 23IX at our institution has the ability to deliver RapidArc plan. Both machines are equipped with kilovoltage cone beam computed tomography (kV-CBCT) and a 120 leaf dynamic millennium multileaf collimator (DMLC).

TABLE 1: Spine SBRT patient data and treatment techniques.

\begin{tabular}{cccc}
\hline \hline Pt. No. & Site & $\begin{array}{c}\text { PTV } \\
\text { Volume }(\mathbf{c c})\end{array}$ & Technique \\
\hline 1 & L4 & 25.5 & 9 Fields-IMRT \\
2 & T7-8 & 100.1 & 9 Fields-IMRT \\
3 & T5 & 40.5 & 9 Fields-IMRT \\
4 & L3-L4 & 83.8 & 9 Fields-IMRT \\
5 & T11-12 & 36.7 & 9 Fields-IMRT \\
\hline \hline
\end{tabular}

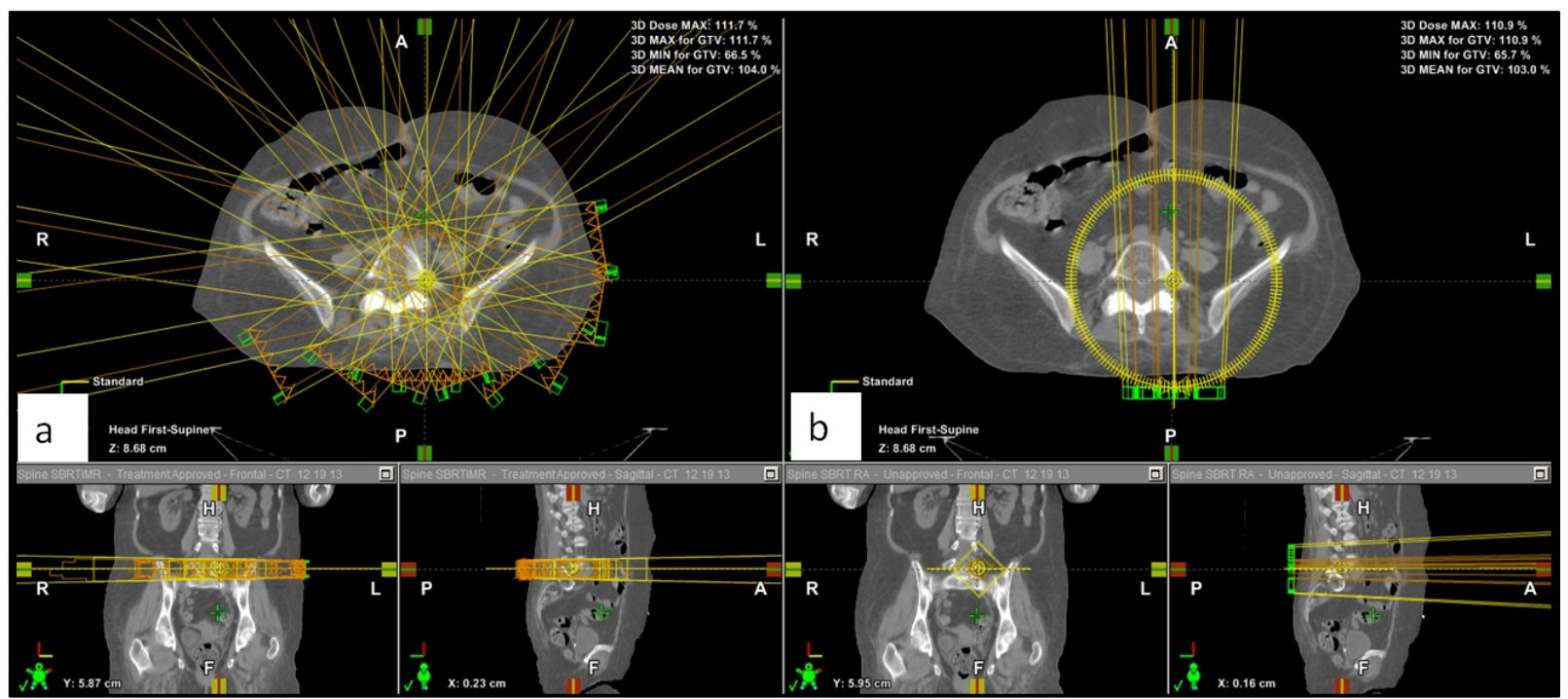

FIG. 1: Beam setup for (a) IMRT plan with 9 beams; and (2) RapidArc with two arcs.

TABLE 2: Planning objectives for IMRT and RapidArc planning.

\begin{tabular}{ll}
\hline \hline \multicolumn{1}{c}{ Volume } & Objective \\
\hline Target & D95\% $=16 \mathrm{~Gy}$ \\
Cord & V10Gy $<10 \%$ \\
& Max $<14 \mathrm{~Gy}$ \\
\hline Maximum dose outside the PTV & $<110 \%$ of the RX dose \\
\hline Dose outside the PTV $+1 \mathrm{~cm}$ & $<105 \%$ of the RX dose \\
\hline \hline
\end{tabular}




\section{Plan evaluation}

Dose volume histograms (DVHs) were evaluated for the target and spinal cord. For the target, the dose to $95 \%$ of the volume (D95\%), the maximum dose to 1cc (Dmax1cc), number of MUs, and the conformity index (CI), and the dose gradient (DG) in centimeters $(\mathrm{cm})$ were assessed. Conformity index is defined by International Commission on Radiation Units and Measurements (ICRU) no. $62{ }^{14}$ as the total volume receiving the prescription dose (cc)/volume of target (cc). Gradient in $\mathrm{cm}$ is defined as the difference between the equivalent sphere radius of the prescription and half-prescription isodoses. Cord maximum dose and volume receiving 10Gy ( $\left.\mathrm{V}_{10 \mathrm{~Gy}}\right)$ were evaluated as well. A quality assurance (QA) was performed for all patients using Portal Dosimetry 10.0 software (Varian Medical systems, Palo Alto, $\mathrm{CA}$ ). The QA images were acquired on amorphous silicon (aSi) EPID. Gamma index values for IMRT and RapidArc were compared. Gamma values such as maximum gamma $\left(\gamma_{\max }\right)$, average gamma $\left(\gamma_{\mathrm{avg}}\right)$, and percentage of the field area with a $\gamma$ value greater than $1.0(\gamma>1)$ were used to evaluate the QA plans.

\section{Results}

\section{Target volume coverage}

For each case, the target volume receiving $100 \%$ of the prescription dose was $95 \%\left(\mathrm{~V}_{100 \%}=95 \%\right)$. The CI, $\mathrm{D}_{\min }(\%), \mathrm{D}_{\max }$ (\%), Dmean (\%), and DG for the target were essentially equivalent. Table 3 summarizes the dosimetric indices of the target. Single factor ANOVA showed no differences between the three groups. Conformal index was not significant among the groups. Figure 2 shows the dose distribution for IMRT and RapidArc for Pt. 1 in Table 1.

TABLE 3: Summary of dosimetric results for the planning target volume (PTV).

\begin{tabular}{cccc}
\hline \hline & IMRT & RapidArc & $P$-value (ANOVA) \\
\hline & $\underline{\text { Average } \pm \text { SD }}$ & $\underline{\text { Average } \pm \text { SD }}$ & \\
$D_{\text {mean }}(\%)$ & $104.1 \pm 1.2$ & $104.1 \pm 1.0$ & 0.9 \\
CI & $1.3 \pm 0.1$ & $1.5 \pm 0.3$ & 0.5 \\
$D_{\max }(\%)$ & $113.1 \pm 5.9$ & $113.6 \pm 5.6$ & 0.9 \\
$D_{\min }(\%)$ & $78.9 \pm 16.3$ & $79.4 \pm 15.7$ & 0.9 \\
$D G(\mathrm{~cm})$ & $1.5 \pm 0.1$ & $1.4 \pm 0.1$ & 0.3 \\
\hline \hline
\end{tabular}

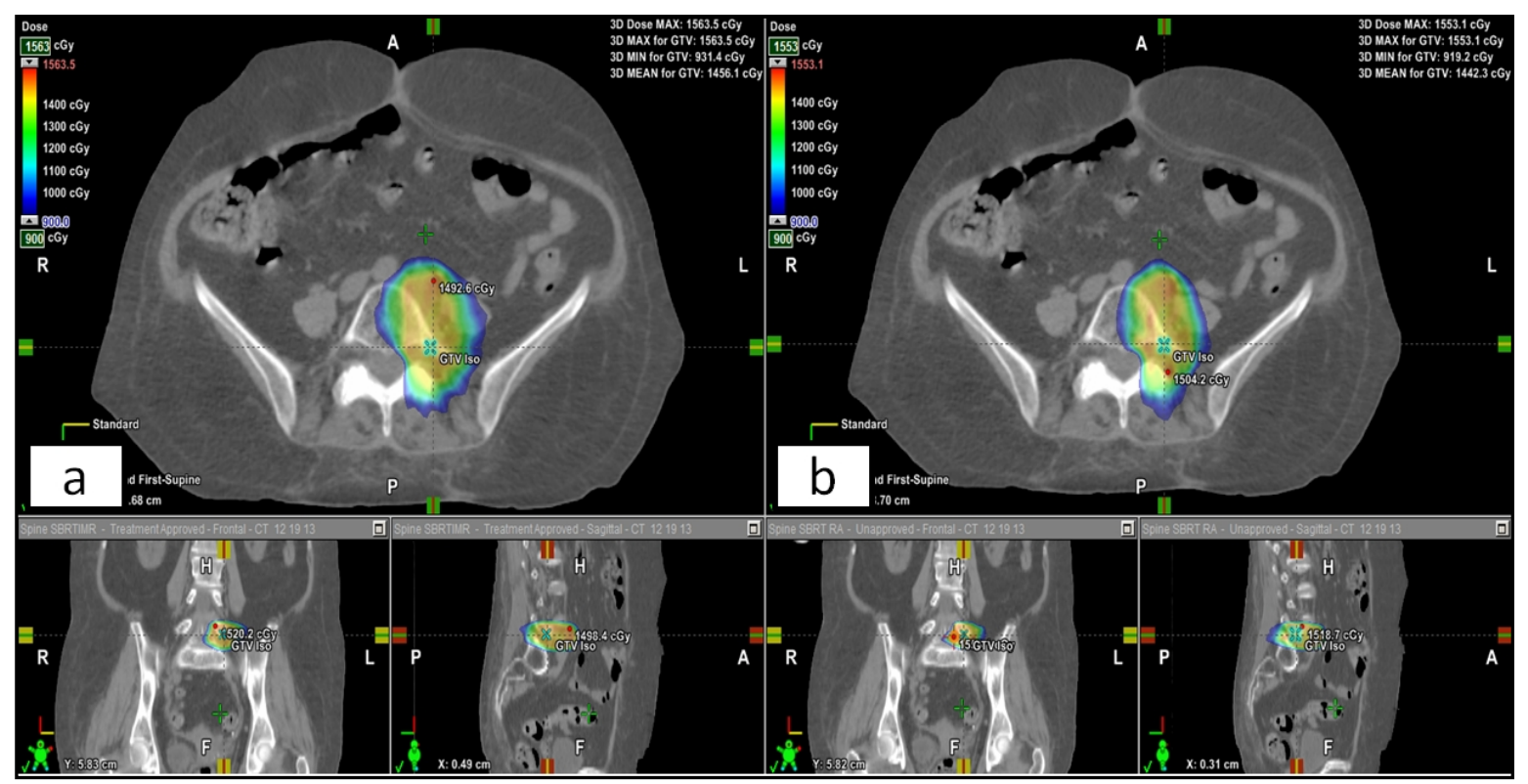

FIG. 2: Dose distribution in color wash for pt. no. 1 in Table 1 (a) IMRT plan; and (b) RapidArc plan.

\section{Spinal cord dose sparing}

Maximum dose to the Cord and the volume receiving $10 \mathrm{~Gy}$ were within the tolerances as recommended by RTOG-0631 and AAPM TG-101. Table 4 summarizes the results.

TABLE 4: Summary of the dosimetric results for spinal cord.

\begin{tabular}{cccc}
\hline \hline & IMRT & RapidArc & $P$-value (ANOVA) \\
\hline Dmax & $11.6 \pm 1.0$ & $11.8 \pm 1.1$ & 0.8 \\
V10Gy & $7.4 \pm 1.4$ & $8.6 \pm 1.7$ & 0.4 \\
\hline \hline
\end{tabular}

TABLE 5: Summary of the average MUs for $2.5 \mathrm{~mm}, 4 \mathrm{~mm}$, and 5 mm MLC leaf width.

\begin{tabular}{ccc}
\hline \hline Technique & Average MU & $P$-value (ANOVA) \\
\hline IMRT & $6771.7 \pm 1323.3$ & 0.02 \\
& & \\
RapidArc & $3978 \pm 576.7$ & \\
\hline \hline
\end{tabular}

Total number of MUs and gamma evaluation

Table 5 summarizes the average number of MUs delivered for each technique. The data shows clearly that the number of MUs using RapidArc technique was reduced significantly 
compare to the IMRT technique $(\mathrm{p}=0.02)$. Gamma evaluation showed a good agreement between the two techniques. The area of gamma $<1.0$ was above $90 \%$ in both techniques.

\section{Discussion}

Spine SBRT requires a delivery of high dose to irregularly shaped target. The dose falloff outside the target necessary should be rapid to avoid any injury to the spinal cord. In this study we investigated the influence of using RapidArc on the plan quality and delivery time as shown in Tables 4 and $\mathbf{5}$. The target coverage, CI, DG, minimum dose, mean dose and maximum dose were equivalent with no significant differences. The spinal cord dosimetric indices were equivalent among the two techniques with no significant differences. The number of MUs was reduced by $\sim 60 \%$ using the RapidArc. As a result of that, the treatment time would be reduced significantly by if RapidArc was used for treatment. Li et al. ${ }^{5}$ reported maximum translational displacements at mid-treatment and post-treatment CBCTs as $3.4 \mathrm{~mm}$ in the lateral direction (vacuum fixation), and $4.5 \mathrm{~mm}$ in the lateral direction (evacuated cushion), respectively. This intrafractional motion can be due to the long treatment time especially for patients with severe back pain so using RapidArc for spine SBRT patients will reduce the treatment time and intrafractional motion significantly.

\section{Conclusion}

The use of RapidArc for planning spine SBRT patients achieved the same plan quality as the IMRT and as recommended by RTOG-0631 and AAPM TG-101. It can also significantly reduce the treatment time and the intrafractional motion. Reduction in treatment time and intrafractional motion can reduce the treatment uncertainties specially for patients presented with severe back pain.

\section{Conflict of interest}

The authors declare that they have no conflicts of interest. The authors alone are responsible for the content and writing of the paper.

\section{References}

1. Sahgal A, Larson DA, Chang EL. Stereotactic body radiosurgery for spinal metastases: a critical review. Int J Radiat Oncol Biol Phys 2008; 71:652-65.
2. Loblaw DA, Laperriere NJ. Emergency treatment of malignant extradural spinal cord compression: an evidence-based guideline. J Clin Oncol 1998; 16:1613-24.

3. Ryu S, Jin R, Jin JY, et al. Pain control by image-guided radiosurgery for solitary spinal metastasis. J Pain Symptom Manage 2008; 35:292-8.

4. Ryu S, Fang Yin F, Rock J, et al. Image-guided and intensity-modulated radiosurgery for patients with spinal metastasis. Cancer 2003; 97:2013-8.

5. Li W, Sahgal A, Foote M, et al. Impact of immobilization on intrafraction motion for spine stereotactic body radiotherapy using cone beam computed tomography. Int J Radiat Oncol Biol Phys 2012; 84:520-6.

6. Otto K. Volumetric modulated arc therapy: IMRT in a single gantry arc. Med Phys 2008; 35:310-7.

7. Cozzi L, Dinshaw KA, Shrivastava SK, et al. A treatment planning study comparing volumetric arc modulation with RapidArc and fixed field IMRT for cervix uteri radiotherapy. Radiother Oncol 2008; 89:180-91.

8. Oliver M, Ansbacher W, Beckham WA. Comparing planning time, delivery time and plan quality for IMRT, RapidArc and Tomotherapy. J Appl Clin Med Phys 2009; 10:3068.

9. Matuszak MM, Yan D, Grills I, Martinez A. Clinical applications of volumetric modulated arc therapy. Int J Radiat Oncol Biol Phys 2010; 77:608-16.

10. Rana S, Rogers K, Pokharel S, Cheng C. Evaluation of Acuros XB algorithm based on RTOG 0813 dosimetric criteria for SBRT lung treatment with RapidArc. J Appl Clin Med Phys 2014; 15:4474.

11. Radiation Therapy Oncology Group RTOG 0631. Phase II/III study of image-guided radiosurgery/SBRT for localized spine metastases; 2009. Available from www.rtog.org

12. Benedict SH, Yenice KM, Followill D, et al. Stereotactic body radiation therapy: the report of AAPM Task Group 101. Med Phys 2010; 37:4078-101.

13. Wu QJ, Yoo S, Kirkpatrick JP, et al. Volumetric arc intensity-modulated therapy for spine body radiotherapy: comparison with static intensity-modulated treatment. Int J Radiat Oncol Biol Phys 2009; 75:1596-604.

14. International Commission on Radiation Units and Measurements ICRU Report 62. Prescribing, recording, and reporting photon beam therapy (Supplement to ICRU Report 50) ICRU, Bethesda, MD, 1999. 